\title{
Multiscale method and pseudospectral simulations for linear viscoelastic incompressible flows
}

\author{
Ling Zhang and Jie Ouyang* \\ Department of Applied Mathematics, Northwestern Polytechnical University, Xi'an, 710129 China
}

(Received May 23, 2011, Revised December 15, 2011, Accepted February 12, 2012)

\begin{abstract}
The two-dimensional incompressible flow of a linear viscoelastic fluid we considered in this research has rapidly oscillating initial conditions which contain both the large scale and small scale information. In order to grasp this double-scale phenomenon of the complex flow, a multiscale analysis method is developed based on the mathematical homogenization theory. For the incompressible flow of a linear viscoelastic Maxwell fluid, a well-posed multiscale system, including averaged equations and cell problems, is derived by employing the appropriate multiple scale asymptotic expansions to approximate the velocity, pressure and stress fields. And then, this multiscale system is solved numerically using the pseudospectral algorithm based on a time-splitting semi-implicit influence matrix method. The comparisons between the multiscale solutions and the direct numerical simulations demonstrate that the multiscale model not only captures large scale features accurately, but also reflects kinetic interactions between the large and small scale of the incompressible flow of a linear viscoelastic fluid.
\end{abstract}

Keywords: linear viscoelastic fluid; incompressible flow; multiscale analysis; averaged equations; cell problems; pseudospectral algorithm.

\section{Introduction}

Various kinds of flows exist widely in physics and engineering, and some flows have multiscale phenomena, for example, the incompressible flow with rapidly oscillating initial data. It is very difficult to solve this kind of multiscale problem by the classical direct numerical simulation (DNS for short) because an accurate solution usually requires very fine meshes and this leads to tremendous amount of computer memory and CPU time. So, it is worthy to develop an effective method of coupling large scales and small scales based on coarse grids, but without solving directly all characteristic quantities on fine grids. In other words, one needs to establish a multiscale model which not only captures the large scale information but also reflects the effects of small scales on large ones. Up to now, there have been some works devoted to developing effective and credible methods, for example, the homogenization method and the multiscale method, to simulate the incompressible flow with rapidly oscillating initial data (McLaughlin et al. 1985, Hou et al. 2004, Hou et al. 2005, Hou et al. 2008).

\footnotetext{
* Corresponding author, Professor, E-mail: jieouyang@nwpu.edu.cn
} 
The earliest attempt was made by McLaughlin, Papanicolaou and Pironneau (MPP for short) in 1985 to research the homogenization of the incompressible Euler equations with rapidly oscillating initial data (McLaughlin et al. 1985). They introduced the mean Lagrangian map and assumed that the oscillation was convected by the mean velocity field so as to construct a multiscale asymptotic expansion for the solution of the Euler equations. Based on the multiscale expansion, a periodic cell problem was obtained for the velocity and pressure fields by assuming that the multiscale solution was periodic in the small scale time and spatial variables. However, the well-posedness of the cell problem was uncertain. So, they imposed the additional assumptions in order to obtain the wellposed equations and then derived a variation of $k-\varepsilon$ model.

In recent years, some researchers have presented the multiscale analysis method for the incompressible flow with the rapidly oscillating initial velocity field (Hou et al. 2004, Hou et al. 2005, Hou et al. 2008). Their key idea in constructing the multiscale solution for the incompressible Euler equations was to reformulate the problem using a new phase variable to characterize the propagation feature of the small scale flow. In virtue of this phase variable the multiscale structure of the solution became apparent. Their analysis was strongly motivated by the pioneering work of MPP; however, the main difference was that they found that the small scale flow was convected not by the mean flow, but by the full velocity field. They thus exactly characterized the nonlinear convection of the small scale flow by a Lagrangian description and turned a convection dominated transport problem into an elliptic problem on a stream function. Then the classical mathematical homogenization theory for elliptic problems (Bensoussan et al. 1978, Oleinik et al. 1992, Jikov et al. 1994, Cioranescu and Donato 1999, Pavliotis and Stuart 2008, Li et al. 2008) can be used to obtain a multiscale asymptotic expansion for the stream function. But since the averaged equation in the Eulerian formulation is more convenient for computations, a coupled homogenized system on the velocity and pressure fields was finally derived according to the key observation from the multiscale analysis in the Lagrangian formulation.

The objects of the aforementioned methods almost focus on Newtonian fluids, while the purpose of this article is to develop the multiscale analysis method for non-Newtonian fluids. The flows of viscoelastic non-Newtonian fluids exist generally in nature and living. We start our studies with a kind of linear viscoelastic non-Newtonian fluid, namely, the incompressible flow of a Maxwell fluid. In comparison with a Newtonian fluid, the governing equations of a non-Newtonian fluid are more complex due to the existence of constitutive equations. Then the effect and evolution of the stress field must be considered in developing the multiscale analysis method for a viscoelastic nonNewtonian fluid. And so it is very difficult and complicated to establish a multiscale model for the incompressible flow of a Maxwell fluid with rapidly oscillating initial data. According to the relation between the velocity and stress field, we expand the stress tensor into a different multiscale asymptotic form from the velocity and pressure fields. Based on these multiscale asymptotic expansions, a multiscale system including the averaged equations and the cell problems is obtained through detailed derivations. However, owing to the complexities of the final averaged equations and cell problems, the numerical simulations become hard and troublesome. In order to solve our multiscale system, the pseudospectral method (Canuto et al. 1988, Shen and Tang 2006, Beris and Dimitropoulos 1999) is used to discretize the governing equations in space, while the integration of the governing equations in time is based on a time-splitting semi-implicit influence matrix method (Beris and Dimitropoulos 1999, Beris and Sureshkumar 1996, Phillips and Soliman 1991). This numerical algorithm ensures not only high precision but also the couplings of velocity-pressure and velocity-stress. It is worth emphasizing that the averaged equations are solved on coarse grids in 
whole domain, while all cell problems are computed in a small periodic subdomain.

The remainder of the paper is organized as follows. The fundamental properties and the governing equations of the 2D incompressible flow of a Maxwell fluid with rapidly oscillating initial data are described in Section 2. Section 3 is devoted to establishing the multiscale model based on the appropriate multiscale asymptotic expansions. And some numerical experiments to verify our multiscale analysis method are carried out in section 4. Conclusions and future research directions conclude the manuscript.

\section{Governing equations}

The 2D incompressible flow of a Maxwell fluid with rapidly oscillating initial data is governed by the following equations

$$
\begin{gathered}
\text { (a) } \nabla \cdot \mathbf{u}^{\varepsilon}=0 \\
\text { (b) } \partial_{t} \mathbf{u}^{\varepsilon}+\mathbf{u}^{\varepsilon} \cdot \nabla \mathbf{u}^{\varepsilon}+\nabla p^{\varepsilon}-\nabla \cdot \mathbf{g}^{\varepsilon}=\mathbf{f} \\
\text { (c) } \lambda \partial_{t} \mathbf{g}^{\varepsilon}+\mathbf{g}^{\varepsilon}=\eta\left[\nabla \mathbf{u}^{\varepsilon}+\left(\nabla \mathbf{u}^{\varepsilon}\right)^{T}\right] \\
\text { (d) } \mathbf{u}^{\varepsilon}(0, \mathbf{x})=\mathbf{U}(\mathbf{x})+\mathbf{W}\left(\mathbf{x}, \frac{\mathbf{x}}{\varepsilon}\right) \\
\text { (e) } \mathbf{g}^{\varepsilon}(0, \mathbf{x})=\eta\left[\nabla \mathbf{u}^{\varepsilon}(0, \mathbf{x})+\left(\nabla \mathbf{u}^{\varepsilon}(0, \mathbf{x})\right)^{T}\right]
\end{gathered}
$$

where $\mathbf{u}^{\varepsilon}$ is the velocity vector, $p^{\varepsilon}$ the pressure, $\mathbf{g}^{\varepsilon}$ the extra-stress tensor, $\mathbf{f}$ a body force, $\lambda$ the relaxation time, $\eta$ the viscosity and $\varepsilon$ is a very small positive number compared with the dimension of macro domain, and the superscript $T$ denotes the transpose of a matrix. In the initial velocity field, $\mathbf{U}(\mathbf{x})$ is assumed to be a smooth mean velocity field and the smooth function $\mathbf{W}(\mathbf{x}, \mathbf{y})$ with $\mathbf{y}=\mathbf{x} / \varepsilon$, which represents the oscillating component of the velocity field, is $\mathbf{y}-$ periodic with a period $2 \pi$ and has zero mean, that is

$$
\langle\mathbf{W}\rangle=\frac{1}{(2 \pi)^{2}} \int_{\Omega} \mathbf{W}(\mathbf{x}, \mathbf{y}) d \mathbf{y}=0
$$

where $\Omega=[0,2 \pi]^{2}$ and the sign $\langle\bullet>$ indicates the space average in $\mathbf{y}$. These conditions mean that the initial velocity field contains both the large sacle and small scale information. The constraints on the small scale component $\mathbf{W}(\mathbf{x}, \mathbf{y})$ are typical hypotheses in the mathematical homogenization theory (Bensoussan et al. 1978).

What concerns us is how to derive the averaged equations on the averaged velocity, pressure and stress fields for a small but finite $\varepsilon$. Actually, the classical mathematical homogenization theory (Bensoussan et al. 1978, Oleinik et al. 1992, Jikov et al. 1994, Cioranescu and Donato 1999, Pavliotis and Stuart 2008, Li et al. 2008) has given us some valuable insights into how the small scale solution interacts with the large one and is propagated in time. So the multiscale analysis method in our research is inspired by the mathematical homogenization theory. However, here the multiscale asymptotic expansions cannot copy the formulas in the classical homogenization method. Otherwise one would not be able to obtain a well-posed cell problem. In addition, how to 
approximate the stress tensor employing a multiscale asymptotic expansion is another difficulty. In order to tackle these problems, we have to look for appropriate multiscale asymptotic expansions.

\section{Multiscale analysis}

The key to multiscale analysis is a proper understanding of how to reflect the evolution on the small scale. Some studies concerning the incompressible flow (Hou et al. 2004, Hou et al. 2005, Hou et al. 2008) have suggested that the small scale solutions are propagated by the Lagrangian map $\theta^{\varepsilon}(t, \mathbf{x})$ which is defined as follows

$$
\partial_{t} \theta^{\varepsilon}+\mathbf{u}^{\varepsilon} \cdot \nabla_{\mathbf{x}} \theta^{\varepsilon}=0, \quad \theta^{\varepsilon}(0, \mathbf{x})=\mathbf{x}
$$

where $\theta^{\varepsilon}(t, \mathbf{x})$ is a Lagrangian coordinate, i.e., the position at time $t$ of the particle transported by the flow $\mathbf{u}^{\varepsilon}$ from position $\mathbf{x}$ at the initial time. A multiscale expansion is employed to approximate the Lagrangian map as

$$
\theta^{\varepsilon}=\bar{\theta}(t, \mathbf{x}, T)+\varepsilon \tilde{\theta}(t, \bar{\theta}, T, \mathbf{y})
$$

where $T=t / \varepsilon, \mathbf{y}=\bar{\theta} / \varepsilon$ are the small scale time and spatial variables respectively. And $\bar{\theta}$ is the average of $\theta^{\varepsilon}$ with respect to $\mathbf{y}$ over one period and $\tilde{\theta}$ is assumed to be a $\mathbf{y}$-periodic function with zero mean. From now on, if the small scale and large scale variables are considered to be independent, then the time and spatial derivatives may be calculated by the chain rule

$$
\begin{array}{r}
\partial_{t} \rightarrow \partial_{t}+\varepsilon^{-1} \partial_{T}+\varepsilon^{-1}\left(\left(\bar{\partial}_{t} \bar{\theta}\right) \cdot \nabla_{\mathbf{y}}\right) \\
\nabla_{\mathbf{x}} \rightarrow \nabla_{\mathbf{x}}+\varepsilon^{-1}\left(\left(\nabla_{\mathbf{x}} \overline{\boldsymbol{\theta}}\right) \cdot \nabla_{\mathbf{y}}\right)
\end{array}
$$

where $\bar{\partial}_{t}=\partial_{t}+\varepsilon^{-1} \partial_{T}$ denotes the total derivative in relation to $t$.

Considering the structure of the initial data and the multiscale expansion of the Lagrangian map as well as the correlation between the velocity and stress field, we employ the multiscale expansions of the velocity, pressure and stress fields with the following forms

$$
\begin{gathered}
\text { (a) } \mathbf{u}^{\varepsilon}(t, \mathbf{x})=\overline{\mathbf{u}}(t, \mathbf{x}, T)+\tilde{\mathbf{u}}(t, \bar{\theta}, T, \mathbf{y}) \\
\text { (b) } p^{\varepsilon}(t, \mathbf{x})=\bar{p}(t, \mathbf{x}, T)+\tilde{p}(t, \bar{\theta}, T, \mathbf{y}) \\
\text { (c) } \mathbf{g}^{\varepsilon}(t, \mathbf{x})=\overline{\mathbf{g}}(t, \mathbf{x}, T)+\varepsilon^{-1} \tilde{\mathbf{g}}(t, \bar{\theta}, T, \mathbf{y})
\end{gathered}
$$

where $\overline{\mathbf{u}}, \bar{p}$ and $\overline{\mathbf{g}}$ are total mean components including higher-order terms, and $\tilde{\mathbf{u}}, \tilde{p}$ and $\tilde{\mathbf{g}}$ are periodic functions and have zero mean with reference to $\mathbf{y}$.

According to the equality $\mathbf{u}^{\varepsilon} \cdot \nabla_{\mathbf{x}} \theta^{\varepsilon}+\left(\nabla_{\mathbf{x}} \cdot \mathbf{u}^{\varepsilon}\right) \theta^{\varepsilon}=\nabla_{\mathbf{x}} \cdot\left(\mathbf{u}^{\varepsilon} \otimes \theta^{\varepsilon}\right)$ and the incompressible condition $\nabla_{\mathbf{x}} \cdot \mathbf{u}^{\varepsilon}=0$, Eq. (3.1) can be rewritten as

$$
\partial_{t} \theta^{\varepsilon}+\nabla_{\mathbf{x}} \cdot\left(\mathbf{u}^{\varepsilon} \otimes \theta^{\varepsilon}\right)=0
$$


Substituting the multiscale expansions Eq. (3.2) and Eq. (3.4 (a)) into Eq. (3.5) yields

$$
\begin{aligned}
\bar{\partial}_{t} \overline{\boldsymbol{\theta}}+\partial_{T} \tilde{\boldsymbol{\theta}}+ & \left(\left(\bar{\partial}_{t} \overline{\boldsymbol{\theta}}\right) \cdot \nabla_{\mathbf{y}}\right) \tilde{\boldsymbol{\theta}}+\varepsilon \partial_{t} \tilde{\boldsymbol{\theta}}+\nabla_{\mathbf{x}} \cdot(\overline{\mathbf{u}} \otimes \overline{\boldsymbol{\theta}}+\varepsilon \overline{\mathbf{u}} \otimes \tilde{\boldsymbol{\theta}}+\tilde{\mathbf{u}} \otimes \overline{\boldsymbol{\theta}}+\varepsilon \tilde{\mathbf{u}} \otimes \tilde{\theta}) \\
& +\varepsilon^{-1}\left(\left(\nabla_{\mathbf{x}} \overline{\boldsymbol{\theta}}\right) \cdot \nabla_{\mathbf{y}}\right) \cdot(\varepsilon \overline{\mathbf{u}} \otimes \tilde{\boldsymbol{\theta}}+\tilde{\mathbf{u}} \otimes \overline{\boldsymbol{\theta}}+\varepsilon \tilde{\mathbf{u}} \otimes \tilde{\theta})=0
\end{aligned}
$$

If we average Eq. (3.6) with respect to $\mathbf{y}$ and apply the periodic and zero mean properties of $\tilde{\theta}$ and $\tilde{\mathbf{u}}$, then the following equation is derived

$$
\bar{\partial}_{t} \bar{\theta}+\nabla_{\mathbf{x}} \cdot(\overline{\mathbf{u}} \otimes \bar{\theta})+\varepsilon \nabla_{\mathbf{x}} \cdot\langle\tilde{\mathbf{u}} \otimes \tilde{\theta}\rangle=0
$$

Subsequently, inserting the multiscale expansions Eq. (3.4) into Eq. (2.1) gives

$$
\begin{gathered}
(a) \nabla_{\mathbf{x}} \cdot(\overline{\mathbf{u}}+\tilde{\mathbf{u}})+\varepsilon^{-1}\left(\left(\nabla_{\mathbf{x}} \bar{\theta}\right) \cdot \nabla_{\mathbf{y}}\right) \cdot \tilde{\mathbf{u}}=0 \\
(b) \bar{\partial}_{t} \overline{\mathbf{u}}+\bar{\partial}_{t} \tilde{\mathbf{u}}+\varepsilon^{-1}\left(\left(\bar{\partial}_{t} \overline{\boldsymbol{\theta}}\right) \cdot \nabla_{\mathbf{y}}\right) \tilde{\mathbf{u}}+\nabla_{\mathbf{x}} \cdot(\overline{\mathbf{u}} \otimes \overline{\mathbf{u}}+\overline{\mathbf{u}} \otimes \tilde{\mathbf{u}}+\tilde{\mathbf{u}} \otimes \overline{\mathbf{u}}+\tilde{\mathbf{u}} \otimes \tilde{\mathbf{u}}) \\
+\varepsilon^{-1}\left(\left(\nabla_{\mathbf{x}} \bar{\theta}\right) \cdot \nabla_{\mathbf{y}}\right) \cdot(\overline{\mathbf{u}} \otimes \tilde{\mathbf{u}}+\tilde{\mathbf{u}} \otimes \overline{\mathbf{u}}+\tilde{\mathbf{u}} \otimes \tilde{\mathbf{u}})+\nabla_{\mathbf{x}} \bar{p}+\nabla_{\mathbf{x}} \tilde{p}+\varepsilon^{-1}\left(\left(\nabla_{\mathbf{x}} \bar{\theta}\right) \cdot \nabla_{\mathbf{y}}\right) \tilde{p} \\
-\nabla_{\mathbf{x}} \cdot \overline{\mathbf{g}}_{-}-\varepsilon^{-1} \nabla_{\mathbf{x}} \cdot \tilde{\mathbf{g}}-\varepsilon^{-2}\left(\left(\nabla_{\mathbf{x}} \bar{\theta}\right) \cdot \nabla_{\mathbf{y}}\right) \cdot \tilde{\mathbf{g}}=\mathbf{f} \\
(c) \lambda \bar{\partial}_{t} \overline{\mathbf{g}}+\varepsilon^{-1} \lambda \bar{\partial}_{t} \tilde{\mathbf{g}}+\varepsilon^{-2} \lambda\left(\left(\bar{\partial}_{t} \overline{\boldsymbol{\theta}}\right) \cdot \nabla_{\mathbf{y}}\right) \tilde{\mathbf{g}}+\overline{\mathbf{g}}+\varepsilon^{-1} \tilde{\mathbf{g}} \\
=\eta\left[\nabla_{\mathbf{x}}(\overline{\mathbf{u}}+\tilde{\mathbf{u}})+\varepsilon^{-1}\left(\left(\nabla_{\mathbf{x}} \bar{\theta}\right) \cdot \nabla_{\mathbf{y}}\right) \tilde{\mathbf{u}}+\left(\nabla_{\mathbf{x}}(\overline{\mathbf{u}}+\tilde{\mathbf{u}})\right)^{T}+\varepsilon^{-1}\left(\left(\left(\nabla_{\mathbf{x}} \bar{\theta}\right) \cdot \nabla_{\mathbf{y}}\right) \tilde{\mathbf{u}}\right)^{T}\right]
\end{gathered}
$$

By taking the average of Eq. (3.8) with regard to $\mathbf{y}$ and making use of the facts that $\tilde{\mathbf{u}}, \tilde{p}$ and $\tilde{\mathbf{g}}$ are periodic and have zero mean, we have

$$
\text { (a) } \nabla_{\mathbf{x}} \cdot \overline{\mathbf{u}}=0
$$

$$
\text { (b) } \bar{\partial}_{t} \overline{\mathbf{u}}+\nabla_{\mathbf{x}} \cdot(\overline{\mathbf{u}} \otimes \overline{\mathbf{u}})+\nabla_{\mathbf{x}} \cdot\langle\tilde{\mathbf{u}} \otimes \tilde{\mathbf{u}}\rangle+\nabla_{\mathbf{x}} \bar{p}-\nabla_{\mathbf{x}} \cdot \overline{\mathbf{g}}=\mathbf{f}
$$

$$
\text { (c) } \lambda \bar{\partial}_{t} \overline{\mathbf{g}}+\overline{\mathbf{g}}=\eta\left[\nabla_{\mathbf{x}} \overline{\mathbf{u}}+\left(\nabla_{\mathbf{x}} \overline{\mathbf{u}}\right)^{T}\right]
$$

where $\langle\tilde{\mathbf{u}} \otimes \tilde{\mathbf{u}}\rangle$ is referred to as the Reynolds stress term. Due to Eq. (3.9) and Eq. (3.8) can be rearranged as

$$
\begin{gathered}
(a) \varepsilon^{-1}\left(\left(\nabla_{\mathbf{x}} \bar{\theta}\right) \cdot \nabla_{\mathbf{y}}\right) \cdot \tilde{\mathbf{u}}+\nabla_{\mathbf{x}} \cdot \tilde{\mathbf{u}}=0 \\
(b) \varepsilon^{-1}\left(\left(\bar{\partial}_{t} \overline{\boldsymbol{\theta}}\right) \cdot \nabla_{\mathbf{y}}\right) \tilde{\mathbf{u}}+\varepsilon^{-1}\left(\left(\nabla_{\mathbf{x}} \bar{\theta}\right) \cdot \nabla_{\mathbf{y}}\right) \cdot(\overline{\mathbf{u}} \otimes \tilde{\mathbf{u}}+\tilde{\mathbf{u}} \otimes \overline{\mathbf{u}}+\tilde{\mathbf{u}} \otimes \tilde{\mathbf{u}}) \\
+\nabla_{\mathbf{x}} \cdot(\overline{\mathbf{u}} \otimes \tilde{\mathbf{u}}+\tilde{\mathbf{u}} \otimes \overline{\mathbf{u}}+\tilde{\mathbf{u}} \otimes \tilde{\mathbf{u}})+\varepsilon^{-1} \partial_{T} \tilde{\mathbf{u}}+\partial_{t} \tilde{\mathbf{u}}-\nabla_{\mathbf{x}} \cdot\langle\tilde{\mathbf{u}} \otimes \tilde{\mathbf{u}}\rangle \\
-\varepsilon^{-2}\left(\left(\nabla_{\mathbf{x}} \bar{\theta}\right) \cdot \nabla_{\mathbf{y}}\right) \cdot \tilde{\mathbf{g}}+\varepsilon^{-1}\left(\left(\nabla_{\mathbf{x}} \bar{\theta}\right) \cdot \nabla_{\mathbf{y}}\right) \tilde{p}-\varepsilon^{-1} \nabla_{\mathbf{x}} \cdot \tilde{\mathbf{g}}+\nabla_{\mathbf{x}} \tilde{p}=0
\end{gathered}
$$




$$
\begin{gathered}
\text { (c) } \varepsilon^{-2} \lambda \partial_{T} \tilde{\mathbf{g}}+\varepsilon^{-2} \lambda\left(\left(\bar{\partial}_{t} \overline{\boldsymbol{\theta}}\right) \cdot \nabla_{\mathbf{y}}\right) \tilde{\mathbf{g}}+\varepsilon^{-1} \lambda \partial_{t} \tilde{\mathbf{g}}+\varepsilon^{-1} \tilde{\mathbf{g}} \\
=\eta\left[\varepsilon^{-1}\left(\left(\nabla_{\mathbf{x}} \overline{\boldsymbol{\theta}}\right) \cdot \nabla_{\mathbf{y}}\right) \tilde{\mathbf{u}}+\varepsilon^{-1}\left(\left(\left(\nabla_{\mathbf{x}} \overline{\boldsymbol{\theta}}\right) \cdot \nabla_{\mathbf{y}}\right) \tilde{\mathbf{u}}\right)^{T}+\nabla_{\mathbf{x}} \tilde{\mathbf{u}}+\left(\nabla_{\mathbf{x}} \tilde{\mathbf{u}}\right)^{T}\right]
\end{gathered}
$$

From Eq. (3.7), Eq. $(3.9(a))$ and Eq. $(3.10(a))$, the first three terms of Eq. $(3.10(b))$ can be simplified as

$$
\begin{gathered}
\varepsilon^{-1}\left(\left(\bar{\partial}_{t} \bar{\theta}\right) \cdot \nabla_{\mathbf{y}}\right) \tilde{\mathbf{u}}+\varepsilon^{-1}\left(\left(\nabla_{\mathbf{x}} \bar{\theta}\right) \cdot \nabla_{\mathbf{y}}\right) \cdot(\overline{\mathbf{u}} \otimes \tilde{\mathbf{u}}+\tilde{\mathbf{u}} \otimes \overline{\mathbf{u}}+\tilde{\mathbf{u}} \otimes \tilde{\mathbf{u}}) \\
+\nabla_{\mathbf{x}} \cdot(\overline{\mathbf{u}} \otimes \tilde{\mathbf{u}}+\tilde{\mathbf{u}} \otimes \overline{\mathbf{u}}+\tilde{\mathbf{u}} \otimes \tilde{\mathbf{u}}) \\
=\varepsilon^{-1}\left(\tilde{\mathbf{u}} \cdot\left(\left(\nabla_{\mathbf{x}} \bar{\theta}\right) \cdot \nabla_{\mathbf{y}}\right)\right) \tilde{\mathbf{u}}+\nabla_{\mathbf{x}} \cdot(\overline{\mathbf{u}} \otimes \tilde{\mathbf{u}})+\tilde{\mathbf{u}} \cdot \nabla_{\mathbf{x}}(\overline{\mathbf{u}}+\tilde{\mathbf{u}})
\end{gathered}
$$

Substituting the equality Eq. (3.11) into Eq. (3.10) results in

$$
\begin{gathered}
(a) \varepsilon^{-1}\left(\left(\nabla_{\mathbf{x}} \overline{\boldsymbol{\theta}}\right) \cdot \nabla_{\mathbf{y}}\right) \cdot \tilde{\mathbf{u}}+\nabla_{\mathbf{x}} \cdot \tilde{\mathbf{u}}=0 \\
(b) \varepsilon^{-1} \partial_{T} \tilde{\mathbf{u}}+\varepsilon^{-1}\left(\tilde{\mathbf{u}} \cdot\left(\left(\nabla_{\mathbf{x}} \overline{\boldsymbol{\theta}}\right) \cdot \nabla_{\mathbf{y}}\right)\right) \tilde{\mathbf{u}}+\nabla_{\mathbf{x}} \cdot(\overline{\mathbf{u}} \otimes \tilde{\mathbf{u}})+\tilde{\mathbf{u}} \cdot \nabla_{\mathbf{x}}(\overline{\mathbf{u}}+\tilde{\mathbf{u}})+\partial_{t} \tilde{\mathbf{u}} \\
-\nabla_{\mathbf{x}} \cdot\langle\tilde{\mathbf{u}} \otimes \tilde{\mathbf{u}}\rangle-\varepsilon^{-2}\left(\left(\nabla_{\mathbf{x}} \overline{\boldsymbol{\theta}}\right) \cdot \nabla_{\mathbf{y}}\right) \cdot \tilde{\mathbf{g}}+\varepsilon^{-1}\left(\left(\nabla_{\mathbf{x}} \overline{\boldsymbol{\theta}}\right) \cdot \nabla_{\mathbf{y}}\right) \tilde{p}-\varepsilon^{-1} \nabla_{\mathbf{x}} \cdot \tilde{\mathbf{g}}+\nabla_{\mathbf{x}} \tilde{p}=0 \\
(c) \varepsilon^{-2} \lambda \partial_{T} \tilde{\mathbf{g}}+\varepsilon^{-2} \lambda\left(\left(\bar{\partial}_{t} \overline{\boldsymbol{\theta}}\right) \cdot \nabla_{\mathbf{y}}\right) \tilde{\mathbf{g}}+\varepsilon^{-1} \lambda \partial_{t} \tilde{\mathbf{g}}+\varepsilon^{-1} \tilde{\mathbf{g}} \\
=\eta\left[\varepsilon^{-1}\left(\left(\nabla_{\mathbf{x}} \overline{\boldsymbol{\theta}}\right) \cdot \nabla_{\mathbf{y}}\right) \tilde{\mathbf{u}}+\varepsilon^{-1}\left(\left(\left(\nabla_{\mathbf{x}} \overline{\boldsymbol{\theta}}\right) \cdot \nabla_{\mathbf{y}}\right) \tilde{\mathbf{u}}\right)^{T}+\nabla_{\mathbf{x}} \tilde{\mathbf{u}}+\left(\nabla_{\mathbf{x}} \tilde{\mathbf{u}}\right)^{T}\right]
\end{gathered}
$$

In order to further simplify our multiscale model, the leading order terms of $(\tilde{\mathbf{u}}, \tilde{p}, \tilde{\mathbf{g}})$ will be researched. To this end $(\tilde{\mathbf{u}}, \tilde{p}, \tilde{\mathbf{g}})$ are expanded as

$$
\begin{aligned}
& \text { (a) } \tilde{\mathbf{u}}=\mathbf{w}(t, \bar{\theta}, T, \mathbf{y})+O(\varepsilon) \\
& \text { (b) } \tilde{p}=q(t, \bar{\theta}, T, \mathbf{y})+O(\varepsilon) \\
& \text { (c) } \tilde{\mathbf{g}}=\tau(t, \bar{\theta}, T, \mathbf{y})+O(\varepsilon)
\end{aligned}
$$

We neglect the $O(\varepsilon)$ terms for the averaged Eq. (3.7) and Eq. (3.9). This implies that

$$
\bar{\partial}_{t} \bar{\theta}+\nabla_{\mathbf{x}} \cdot(\overline{\mathbf{u}} \otimes \bar{\theta})=0
$$

and

$$
\text { (a) } \nabla_{\mathbf{x}} \cdot \overline{\mathbf{u}}=0
$$

(b) $\bar{\partial}_{t} \overline{\mathbf{u}}+\nabla_{\mathbf{x}} \cdot(\overline{\mathbf{u}} \otimes \overline{\mathbf{u}})+\nabla_{\mathbf{x}} \cdot\langle\mathbf{w} \otimes \mathbf{w}\rangle+\nabla_{\mathbf{x}} \bar{p}-\nabla_{\mathbf{x}} \cdot \overline{\mathbf{g}}=\mathbf{f}$

$$
\text { (c) } \lambda \bar{\partial}_{t} \overline{\mathbf{g}}+\overline{\mathbf{g}}=\eta\left[\nabla_{\mathbf{x}} \overline{\mathbf{u}}+\left(\nabla_{\mathbf{x}} \overline{\mathbf{u}}\right)^{T}\right]
$$

Multiplying both sides of Eq. (3.12) by $\varepsilon$ yields 


$$
\begin{gathered}
(a)\left(\left(\nabla_{\mathbf{x}} \overline{\boldsymbol{\theta}}\right) \cdot \nabla_{\mathbf{y}}\right) \cdot \tilde{\mathbf{u}}+O(\varepsilon)=0 \\
\text { (b) } \partial_{T} \tilde{\mathbf{u}}+\left(\tilde{\mathbf{u}} \cdot\left(\left(\nabla_{\mathbf{x}} \overline{\boldsymbol{\theta}}\right) \cdot \nabla_{\mathbf{y}}\right)\right) \tilde{\mathbf{u}}+\left(\left(\nabla_{\mathbf{x}} \overline{\boldsymbol{\theta}}\right) \cdot \nabla_{\mathbf{y}}\right) \tilde{p} \\
-\varepsilon^{-1}\left(\varepsilon \nabla_{\mathbf{x}}+\left(\nabla_{\mathbf{x}} \overline{\boldsymbol{\theta}}\right) \cdot \nabla_{\mathbf{y}}\right) \cdot \tilde{\mathbf{g}}+O(\varepsilon)=0 \\
(c) \varepsilon^{-1} \lambda\left(\varepsilon \partial_{t}+\partial_{T}\right) \tilde{\mathbf{g}}+\varepsilon^{-1} \lambda\left(\left(\bar{\partial}_{t} \overline{\boldsymbol{\theta}}\right) \cdot \nabla_{\mathbf{y}}\right) \tilde{\mathbf{g}}+\tilde{\mathbf{g}} \\
=\eta\left[\left(\left(\nabla_{\mathbf{x}} \overline{\boldsymbol{\theta}}\right) \cdot \nabla_{\mathbf{y}}\right) \tilde{\mathbf{u}}+\left(\left(\left(\nabla_{\mathbf{x}} \overline{\boldsymbol{\theta}}\right) \cdot \nabla_{\mathbf{y}}\right) \tilde{\mathbf{u}}\right)^{T}\right]+O(\varepsilon)
\end{gathered}
$$

To the leading order approximation, we neglect the $O(\varepsilon)$ terms associated with the divergence operator in the momentum Eq. $(3.16(b))$ and the time derivative operator in the constitutive Eq. $(3.16(c))$, and then the following equation is obtained

$$
\text { (a) }\left(\left(\nabla_{\mathbf{x}} \overline{\boldsymbol{\theta}}\right) \cdot \nabla_{\mathbf{y}}\right) \cdot \mathbf{w}=0
$$

$$
\begin{gathered}
\text { (b) } \partial_{T} \mathbf{w}+\left(\mathbf{w} \cdot\left(\left(\nabla_{\mathbf{x}} \bar{\theta}\right) \cdot \nabla_{\mathbf{y}}\right)\right) \mathbf{w}+\left(\left(\nabla_{\mathbf{x}} \bar{\theta}\right) \cdot \nabla_{\mathbf{y}}\right) q-\varepsilon^{-1}\left(\left(\nabla_{\mathbf{x}} \bar{\theta}\right) \cdot \nabla_{\mathbf{y}}\right) \cdot \tau=0 \\
\text { (c) } \varepsilon^{-1} \lambda \partial_{T} \tau+\varepsilon^{-1} \lambda\left(\left(\bar{\partial}_{t} \bar{\theta}\right) \cdot \nabla_{\mathbf{y}}\right) \tau+\tau=\eta\left[\left(\left(\nabla_{\mathbf{x}} \bar{\theta}\right) \cdot \nabla_{\mathbf{y}}\right) \mathbf{w}+\left(\left(\left(\nabla_{\mathbf{x}} \bar{\theta}\right) \cdot \nabla_{\mathbf{y}}\right) \mathbf{w}\right)^{T}\right]
\end{gathered}
$$

To sum up, considering the corresponding initial conditions, we get the following multiscale model:

(1) Averaged equations for the leading order velocity, pressure and stress fields

$$
\text { (a) } \nabla_{\mathbf{x}} \cdot \overline{\mathbf{u}}=0
$$

(b) $\bar{\partial}_{t} \overline{\mathbf{u}}+\nabla_{\mathbf{x}} \cdot(\overline{\mathbf{u}} \otimes \overline{\mathbf{u}})+\nabla_{\mathbf{x}} \cdot\langle\mathbf{w} \otimes \mathbf{w}\rangle+\nabla_{\mathbf{x}} \bar{p}-\nabla_{\mathbf{x}} \cdot \overline{\mathbf{g}}=\mathbf{f}$

$$
\begin{gathered}
\text { (c) } \lambda \overline{\partial_{t}} \overline{\mathbf{g}}+\overline{\mathbf{g}}=\eta\left[\nabla_{\mathbf{x}} \overline{\mathbf{u}}+\left(\nabla_{\mathbf{x}} \overline{\mathbf{u}}\right)^{T}\right] \\
\text { (d) }\left.\overline{\mathbf{u}}\right|_{t=0}=\mathbf{U}(\mathbf{x}) \\
\text { (e) }\left.\overline{\mathbf{g}}\right|_{t=0}=\eta\left[\nabla_{\mathbf{x}} \mathbf{U}+\left(\nabla_{\mathbf{x}} \mathbf{U}\right)^{T}\right]
\end{gathered}
$$

(2) Averaged equations for the leading order Lagrangian map

$$
\begin{gathered}
\text { (a) } \overline{\partial_{t}} \bar{\theta}+\nabla_{\mathbf{x}} \cdot(\overline{\mathbf{u}} \otimes \bar{\theta})=0 \\
\text { (b) }\left.\overline{\boldsymbol{\theta}}\right|_{t=0}=\mathbf{x}
\end{gathered}
$$

(3) Leading order equations for the fluctuations of the velocity, pressure and stress fields

$$
\text { (a) }\left(\left(\nabla_{\mathbf{x}} \bar{\theta}\right) \cdot \nabla_{\mathbf{y}}\right) \cdot \mathbf{w}=0
$$

(b) $\partial_{T} \mathbf{w}+\left(\mathbf{w} \cdot\left(\left(\nabla_{\mathbf{x}} \bar{\theta}\right) \cdot \nabla_{\mathbf{y}}\right)\right) \mathbf{w}+\left(\left(\nabla_{\mathbf{x}} \bar{\theta}\right) \cdot \nabla_{\mathbf{y}}\right) q-\varepsilon^{-1}\left(\left(\nabla_{\mathbf{x}} \bar{\theta}\right) \cdot \nabla_{\mathbf{y}}\right) \cdot \tau=0$ 


$$
\begin{aligned}
&(c) \varepsilon^{-1} \lambda \partial_{T} \tau+\varepsilon^{-1} \lambda\left(\left(\bar{\partial}_{t} \bar{\theta}\right) \cdot \nabla_{\mathbf{y}}\right) \tau+\tau=\eta\left[\left(\left(\nabla_{\mathbf{x}} \overline{\boldsymbol{\theta}}\right) \cdot \nabla_{\mathbf{y}}\right) \mathbf{w}+\left(\left(\left(\nabla_{\mathbf{x}} \overline{\boldsymbol{\theta}}\right) \cdot \nabla_{\mathbf{y}}\right) \mathbf{w}\right)^{T}\right] \\
&\left.(d) \mathbf{w}\right|_{T=t=0}=\mathbf{W}(\mathbf{x}, \mathbf{y}) \\
&\left(\text { e) }\left.\tau\right|_{T=t=0}=\eta\left[\nabla_{\mathbf{y}} \mathbf{W}+\left(\nabla_{\mathbf{y}} \mathbf{W}\right)^{T}\right]\right.
\end{aligned}
$$

The averaged Eq. (3.18) with respect to $(\overline{\mathbf{u}}, \bar{p}, \overline{\mathbf{g}})$ and the cell problem Eq. (3.20) with regard to $(\mathbf{w}, q, \tau)$ plus the averaged Eq. (3.19) with respect to $\bar{\theta}$ constitute the multiscale model of the incompressible flow of a linear viscoelastic fluid. By contrast with the corresponding Newtonian fluids (Hou et al. 2004, Hou et al. 2005, Hou et al. 2008), this multiscale model is more complex owing to the evolution of the stress field, and it is more difficult to solve these equations numerically. Moreover, it is found that the averaged Eq. (3.18) describes the incompressible flow of a Maxwell fluid driven by a force, while the cell problem Eq. (3.20) is relatively intricate.

\section{Numerical experiments}

In this section, the computational results obtained from the multiscale system are compared with the direct numerical simulations for the purpose of checking the validity and accuracy of the multiscale analysis method.

\subsection{Numerical algorithm}

In the following, the numerical algorithm of multiscale simulations is described. The averaged equations are solved on a coarse grid with a large time-step $\Delta t$, while a subgrid space-step $\Delta \mathbf{y}$ and a subgrid time-step $\Delta T$ are adopted to solve the cell problems with the periodic boundary condition in y. For an integer $m \geq 0$, let $t_{m}=m \Delta t$ and $T_{m}=m \Delta t / \varepsilon$. Define $\Delta=\Delta t / \varepsilon$ and $[f]^{*}$ to be the local time average given by

$$
\left[f^{m}\right]_{\Delta}^{*}=\frac{1}{\Delta} \int_{T_{m-1}}^{T_{m}} f d T
$$

Furthermore, we note that the large scale solutions $(\overline{\mathbf{u}}, \bar{p}, \overline{\mathbf{g}})$ and $\bar{\theta}$ have the following forms

$$
\begin{aligned}
& \text { (a) } \overline{\mathbf{u}}(t, \mathbf{x}, T)=\mathbf{u}(t, \mathbf{x})+\varepsilon \mathbf{u}_{1}(t, \mathbf{x}, T) \\
& \text { (b) } \bar{p}(t, \mathbf{x}, T)=p(t, \mathbf{x})+\varepsilon p_{1}(t, \mathbf{x}, T) \\
& \text { (c) } \overline{\mathbf{g}}(t, \mathbf{x}, T)=\mathbf{g}(t, \mathbf{x})+\varepsilon \mathbf{g}_{1}(t, \mathbf{x}, T) \\
& \text { (d) } \bar{\theta}(t, \mathbf{x}, T)=\theta(t, \mathbf{x})+\varepsilon \theta_{1}(t, \mathbf{x}, T)
\end{aligned}
$$

Here we focus on the leading order approximation of the large scale solutions and then the following algorithm is obtained in detail:

Step 1. (Set the initial conditions) At $t=0$ and $T=0$, we set the initial conditions 


$$
\begin{gathered}
\theta_{\text {init }}=\mathbf{x}, \mathbf{u}_{\text {init }}=\mathbf{U}, \mathbf{g}_{\text {init }}=\eta\left(\nabla_{\mathbf{x}} \mathbf{U}+\left(\nabla_{\mathbf{x}} \mathbf{U}\right)^{T}\right) \\
\mathbf{w}_{\text {init }}=\mathbf{W}, \boldsymbol{\tau}_{\text {init }}=\eta\left(\nabla_{\mathbf{y}} \mathbf{W}+\left(\nabla_{\mathbf{y}} \mathbf{W}\right)^{T}\right)
\end{gathered}
$$

Then start the iteration for $m=0,1, \ldots$ as follows:

Step 2. (Update the small scale solutions) Solve all cell problems for $T_{m}<T \leq T_{m+1}$ where every cell solution $(\mathbf{w}, q, \tau)$ satisfies the following equation while keeping $\mathbf{x}$ and $t$ fixed as parameters

$$
\text { (a) }\left(\left(\nabla_{\mathbf{x}} \theta\right) \cdot \nabla_{\mathbf{y}}\right) \cdot \mathbf{w}=0
$$

(b) $\partial_{T} \mathbf{w}+\left(\mathbf{w} \cdot\left(\left(\nabla_{\mathbf{x}} \theta\right) \cdot \nabla_{\mathbf{y}}\right)\right) \mathbf{w}+\left(\left(\nabla_{\mathbf{x}} \theta\right) \cdot \nabla_{\mathbf{y}}\right) q-\varepsilon^{-1}\left(\left(\nabla_{\mathbf{x}} \theta\right) \cdot \nabla_{\mathbf{y}}\right) \cdot \tau=0$

(c) $\varepsilon^{-1} \lambda \partial_{T} \tau+\varepsilon^{-1} \lambda\left(\left(\partial_{t} \theta\right) \cdot \nabla_{\mathbf{y}}\right) \tau+\tau=\eta\left[\left(\left(\nabla_{\mathbf{x}} \theta\right) \cdot \nabla_{\mathbf{y}}\right) \mathbf{w}+\left(\left(\left(\nabla_{\mathbf{x}} \theta\right) \cdot \nabla_{\mathbf{y}}\right) \mathbf{w}\right)^{T}\right]$

$$
\text { (d) }\left.\mathbf{w}\right|_{T=t=0}=\mathbf{W}(\mathbf{x}, \mathbf{y})
$$

$$
\text { (e) }\left.\tau\right|_{T=t=0}=\eta\left[\nabla_{\mathbf{y}} \mathbf{W}+\left(\nabla_{\mathbf{y}} \mathbf{W}\right)^{T}\right]
$$

It is worth noting that the coefficients of this equation vary with the location of cells, that is to say, every cell may have the different solution.

Step 3. (Evaluate the Reynolds stress) Compute $\left\langle[\mathbf{w} \otimes \mathbf{w}]_{\Delta}^{*}\right\rangle$ for every cell, that is, firstly integrate $\mathbf{w} \otimes \mathbf{w}$ with respect to $T$ over $\Delta$ to get $[\mathbf{w} \otimes \mathbf{w}]_{\Delta}^{*}$, and then integrate $[\mathbf{w} \otimes \mathbf{w}]_{\Delta}^{*}$ with regard to $\mathbf{y}$ over $[0,2 \pi]^{2}$ to obtain $\left\langle[\mathbf{w} \otimes \mathbf{w}]_{\Delta}^{*}\right\rangle$.

Step 4. (Update the large scale solutions) Solve $(\mathbf{u}, p, \mathbf{g})$ and $\theta$ for $t_{m}<t \leq t_{m+1}$ which are governed by the following system

$$
\text { (a) } \nabla_{\mathbf{x}} \cdot \mathbf{u}=0
$$

(b) $\partial_{t} \mathbf{u}+\mathbf{u} \cdot \nabla_{\mathbf{x}} \mathbf{u}+\nabla_{\mathbf{x}} \cdot\left\langle[\mathbf{w} \otimes \mathbf{w}]_{\Delta}^{*}\right\rangle+\nabla_{\mathbf{x}} p-\nabla_{\mathbf{x}} \cdot \mathbf{g}=\mathbf{f}$

(c) $\lambda \partial_{t} \mathbf{g}+\mathbf{g}=\eta\left[\nabla_{\mathbf{x}} \mathbf{u}+\left(\nabla_{\mathbf{x}} \mathbf{u}\right)^{T}\right]$

$$
\text { (d) }\left.\mathbf{u}\right|_{t=0}=\mathbf{U}(\mathbf{x})
$$

(e) $\left.\mathbf{g}\right|_{t=0}=\eta\left[\nabla_{\mathbf{x}} \mathbf{U}+\left(\nabla_{\mathbf{x}} \mathbf{U}\right)^{T}\right]$

and

$$
\text { (a) } \partial_{t} \boldsymbol{\theta}+\mathbf{u} \cdot \nabla_{\mathbf{x}} \boldsymbol{\theta}=0
$$

$$
\text { (b) }\left.\boldsymbol{\theta}\right|_{t=0}=\mathbf{x}
$$

Step 5. (Reset the initial conditions for the next time) Let

$$
\begin{gathered}
\theta_{\text {init }}=\left.\boldsymbol{\theta}\right|_{t=t_{m+1}}, \mathbf{u}_{\text {init }}=\left.\mathbf{u}\right|_{t=t_{m+1}}, \mathbf{g}_{\text {init }}=\left.\mathbf{g}\right|_{t=t_{m+1}} \\
\mathbf{w}_{\text {init }}=\left.\mathbf{w}\right|_{T=T_{m+1}}, \boldsymbol{\tau}_{\text {init }}=\left.\boldsymbol{\tau}\right|_{T=T_{m+1}}
\end{gathered}
$$


Repeat Step 2 to Step 5 until the final time arrives.

According to the above algorithm procedure, the multiscale model is tested in a doubly periodic box with size of $2 \pi \times 2 \pi$. Then the original governing Eq. (2.1) and the averaged Eq. (4.5) satisfy the periodic boundary conditions. In view of the periodicity the Fourier pseudospectral method (Canuto et al. 1988, Shen and Tang 2006, Beris and Dimitropoulos 1999) is used to discretize the governing equations in space. In order to eliminate the aliasing error in the pseudospectral method, a 16th order Fourier smoothing function is employed to damp down the high frequency modes (Shen and Tang 2006, Hou and Li 2006). And the integration of the governing equations in time is based on a time-splitting semi-implicit influence matrix method (Beris and Dimitropoulos 1999, Beris and Sureshkumar 1996, Phillips and Soliman 1991). In particular, a second order AdamsBashforth method is used for the explicit update of the non-linear terms and a second order AdamsMoulton scheme for the implicit update of the linear terms (Beris and Dimitropoulos 1999, Beris and Sureshkumar 1996).

We employ the averaged solutions and the cell solutions to reconstruct the fine grid velocity, pressure and stress fields for the purpose of comparing with the DNS. That is

$$
\begin{gathered}
\mathbf{u}^{\varepsilon}(t, \mathbf{x}) \approx \mathbf{u}(t, \mathbf{x})+\mathbf{w}(t, \theta(t, \mathbf{x}), T, \theta(t, \mathbf{x}) / \varepsilon) \\
p^{\varepsilon}(t, \mathbf{x}) \approx p(t, \mathbf{x})+q(t, \theta(t, \mathbf{x}), T, \theta(t, \mathbf{x}) / \varepsilon) \\
\mathbf{g}^{\varepsilon}(t, \mathbf{x}) \approx \mathbf{g}(t, \mathbf{x})+\varepsilon^{-1} \tau(t, \theta(t, \mathbf{x}), T, \theta(t, \mathbf{x}) / \varepsilon)
\end{gathered}
$$

where the mean Lagrangian map in fine grid is obtained by the spectral interpolation (Shen and Tang 2006).

\subsection{Numerical results}

In our numerical simulations, the value of viscosity is taken as $\eta=0.1$ and the relaxation time is $\lambda=0.1$. And the parameter $\varepsilon$ is chosen as $\varepsilon=1 / 32$, then the domain of $[0,2 \pi]^{2}$ is divided into $32 \times 32$ cells. For the averaged equations the coarse mesh resolution is $64 \times 64$ along $x_{1}, x_{2}$, respectively and the time-step is $\Delta t=0.02$, while a mesh size of $32 \times 32$ and the subgrid timestep $\Delta T=0.02$ are used to solve the cell problems. In addition the DNS uses a $512 \times 512$ mesh.

We choose the initial velocity fields as the following forms

$$
\begin{array}{ll}
U_{1}(\mathbf{x})=-\sin \left(x_{1}\right) \cos \left(x_{2}\right), & U_{2}(\mathbf{x})=\cos \left(x_{1}\right) \sin \left(x_{2}\right) \\
W_{1}(\mathbf{x}, \mathbf{x} / \varepsilon)=\sin \left(x_{2} / \varepsilon\right), & W_{2}(\mathbf{x}, \mathbf{x} / \varepsilon)=\sin \left(x_{1} / \varepsilon\right)
\end{array}
$$

Then the initial stress distributions can be obtained according to the initial velocity field and Eq. $(2.1(e))$. The multiscale system and the original governing equations are solved numerically in terms of these initial conditions. Fig. 1 gives the contour plots of the velocity and vorticity distributions at $t=0.8$, and those of the normal stress difference $g_{11}^{\varepsilon}-g_{22}^{\varepsilon}$, shear stress $g_{12}^{\varepsilon}$ and pressure field are shown in Fig. 2, where the left plots represent the DNSs and the right plots represent the multiscale simulations. As shown in Fig. 1 and Fig. 2, the multiscale simulations are basically accordant with the DNSs; especially good agreements are received for the velocity, vorticity and pressure distributions. In view of Eq. (4.8), it is found that the approximation on the 

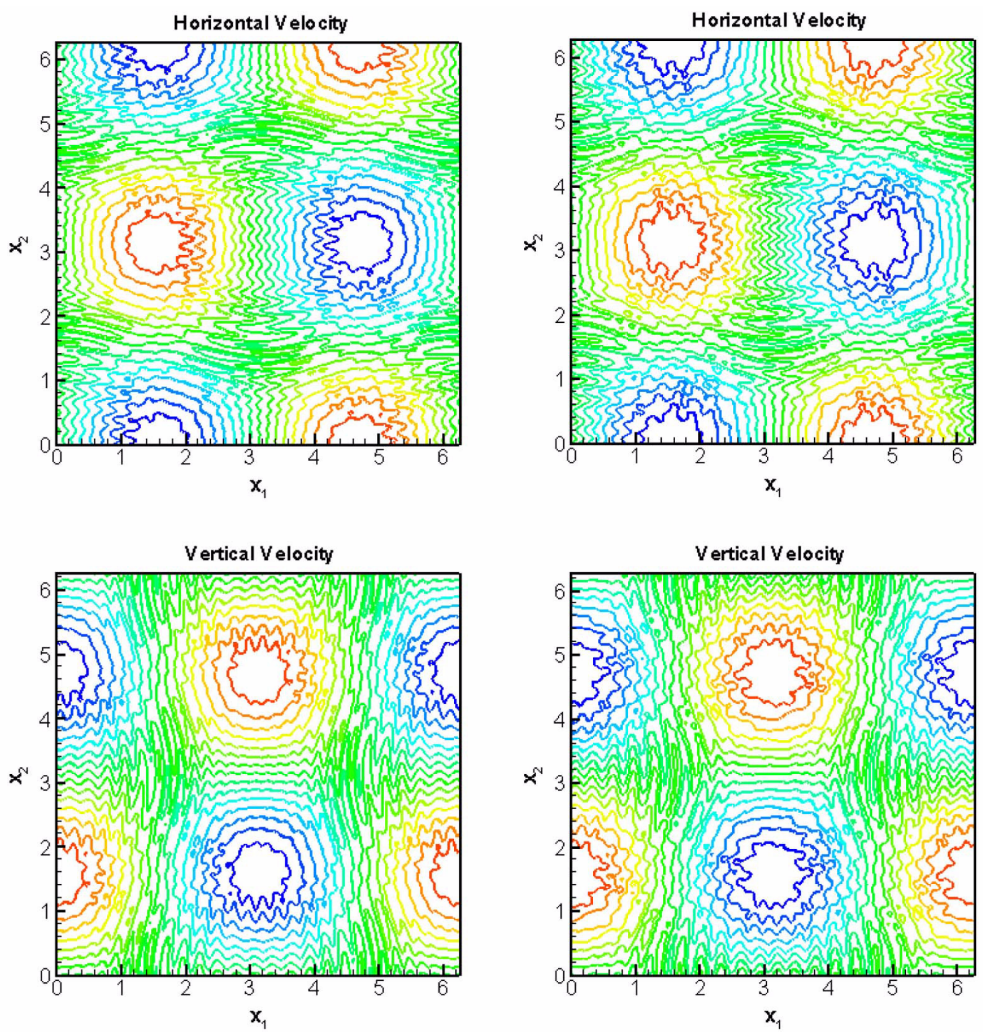

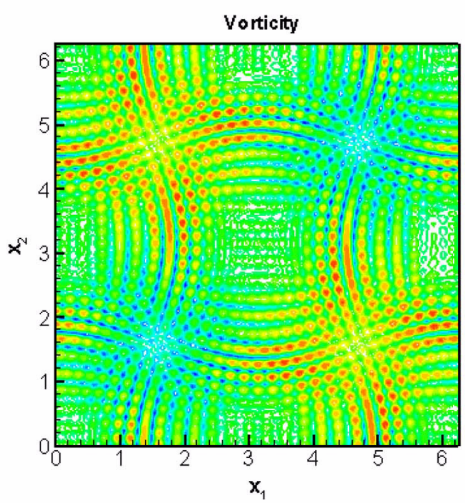

(a) DNS

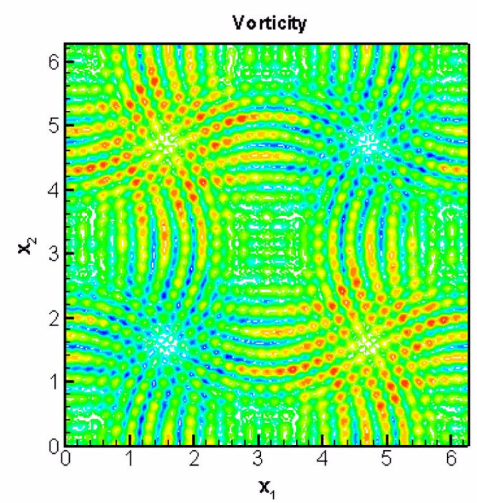

(b) Multiscale solution

Fig. 1 Contour plots of velocity field and vorticity at $t=0.8$

stress has low order compared to those of the approximations on the velocity and pressure, so the differences between the multiscale simulations and the DNSs are more apparent for the normal stress difference and the shear stress. In addition, the solutions tend to be more and more stable over time, and the changes of the contour plots are getting smaller and smaller. These comparisons and analyses indicate that the multiscale model not only captures the large scale features of the 

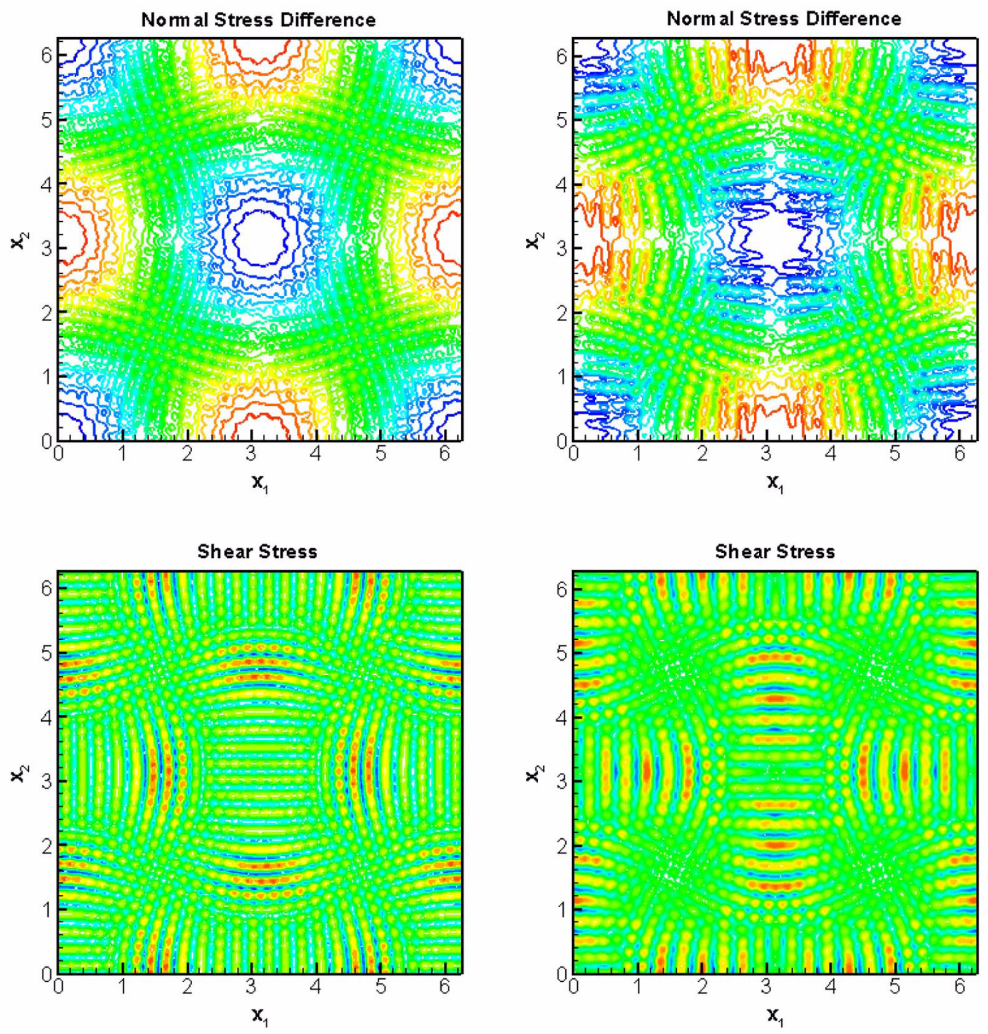

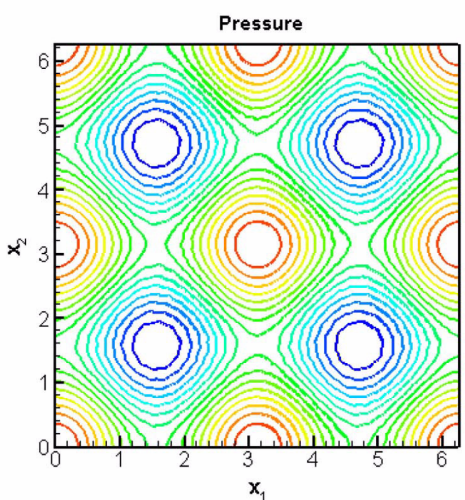

(a) DNS

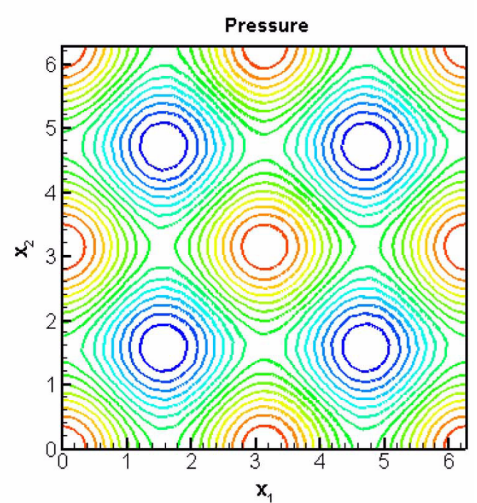

(b) Multiscale solution

Fig. 2 Contour plots of normal stress difference, shear stress and pressure field at $t=0.8$

incompressible flow of a linear viscoelastic non-Newtonian fluid, but also reflects the kinetic interactions between the large scale and small one.

Some different simulations were carried out for the different initial conditions and parameters $(\eta, \lambda, \varepsilon)$, especially for the different $\varepsilon$. It is found that the initial conditions and the parameters $(\eta, \lambda)$ have little effect on the difference between the multiscale simulations and DNSs, while the 
smaller the parameter $\varepsilon$ is, the smaller differences between two methods have become. But the value of $\varepsilon$ is determined by the computational grid that we will use to solve the large scale solution, and the amount of computation increases as the parameter $\varepsilon$ decreases. Therefore, the appropriate $\varepsilon$ is chosen in our simulation.

\section{Conclusions}

For the incompressible flow of a Maxwell fluid with rapidly oscillating initial data, we introduce the Lagrangian map and make the appropriate multiscale asymptotic expansions in the Eulerian formulation, and then a multiscale system, which includes the averaged equations in the large scale and the cell problems in the small scale, is established. The multiscale system and the original governing equations are solved accurately by the pseudospectral algorithm based on a time-splitting semi-implicit influence matrix method. The agreements between the multiscale solutions and the DNSs indicate that the multiscale model not only captures the large scale features of flows, but also reflects the interactions between the large and small scale. Therefore, our multiscale analysis method is effective and feasible for the incompressible flow of a linear viscoelastic non-Newtonian fluid.

In the present work, we note that the difference between the multiscale solutions and DNSs becomes more and more obvious when long time calculations are performed. The reason may be the excessive deformation in the Lagrangian map. Then we need to look for the appropriate technique to avoid this kind of deformation. Moreover, if initial data have many non-separable scales, then how to develop a multiscale analysis method is a very significant issue. Furthermore, extending the multiscale analysis method to non-linear viscoelastic non-Newtonian fluids is more important and required. These are just few of the issues that will be investigated in our future work.

\section{Acknowledgements}

The financial supports of the Natural Sciences Foundation in China under grants 10590353, 10871159 and the Special Funds for Major State Basic Research Projects under grant 2005CB321704 are gratefully acknowledged.

\section{References}

Bensoussan, A., Lions, J.L. and Papanicolaou, G. (1978), Asymptotic analysis for periodic structures, NorthHolland Publishing Company, Netherlands.

Beris, A.N. and Dimitropoulos, C.D. (1999), "Pseudospectral simulation of turbulent viscoelastic channel flow", Comput. Method. Appl. M., 180(3-4), 365-392.

Beris, A.N. and Sureshkumar, R. (1996), "Simulation of time-dependent viscoelastic channel poiseuille flow at high reynolds numbers", Chem. Eng. Sci., 51(9), 1451-1471.

Canuto, C., Hussaini, M.Y., Quarteroni, A. and Zang, T.A. (1988), Spectral methods in fluid dynamics, SpringerVerlag, New York.

Cioranescu, D. and Donato, P. (1999), An introduction to homogenization, Oxford University Press, Oxford.

Hou, T.Y. and Li, R. (2006), "Dynamic depletion of vortex stretching and non-blowup of the 3-D incompressible Euler equations", J. Nonlinear Sci., 16, 639-664. 
Hou, T.Y., Yang, D.P. and Ran, H.Y. (2005), "Multiscale analysis in Lagrangian formulation for the 2D incompressible Euler equation”, Discrete Cont. Dyn. S., 13(5), 1153-1186.

Hou, T.Y., Yang, D.P. and Ran, H.Y. (2008), "Multi-scale analysis and computation for the 3D incompressible Navier-Stokes equations", Multiscale Model Sim., 4, 1317-1346.

Hou, T.Y., Yang, D.P. and Wang, K. (2004), "Homogenization of incompressible Euler equation", J. Comput. Math, 22(2), 220-229.

Jikov, V., Kozlov, S.M. and Oleinik, O.A. (1994), Homogenization of differential operators and integral functionals, Springer-Verlag, Berlin.

Li, A., Li, R. and Fish, J. (2008), "Generalized mathematical homogenization: from theory to practice", Comput. Method. Appl. M., 197(41-42), 3225-3248.

McLaughlin, D.W., Papanicolaou, G.C. and Pironneau, O.R. (1985), "Convection of microstructure and related problems", SIAM J. Appl. Math., 45(5), 780-797.

Oleinik, O.A., Shamaev, A.S. and Yosifian, G.A. (1992), Mathematical problems in elasticity and homogenization, North-Holland Publishing Company, Netherlands.

Pavliotis, G.A. and Stuart, A.M. (2008), Multiscale methods: averaging and homogenization, Texts in Applied Mathematics, 53, Springer, New York.

Phillips, T.N. and Soliman, I.M. (1991), "Influence matrix technique for the numerical spectral simulation of viscous incompressible flows", Numer. Meth. Part. D. E., 7(1), 9-24.

Shen, J. and Tang, T. (2006), Spectral and high-order methods with applications, Science Press, Beijing. 